\title{
Effect of long slow distance exercise regime on health- related physical fitness components among overweight adults with non-communicable diseases
}

\author{
Mastura Johar1*, Tan Chin Hung1', Haizan Mohd Taha', Amira Raihana Adnan², Muhamad \\ Safwan Ishak ${ }^{2}$, Alicia Philip ${ }^{2}$ and Mohd Nahar Azmi ${ }^{3}$ \\ ${ }^{1}$ College of Engineering, Universiti Tenaga Nasional, Malaysia. \\ ${ }^{2}$ College of Foundation \& Diploma Studies, Universiti Tenaga Nasional, Malaysia. \\ ${ }^{3}$ Faculty Medicine, University Malaya, Malaysia.
}

Accepted 23 November, 2018

\begin{abstract}
The purpose of this study was to evaluate the effect of a 12-weeks of long slow distance (LSD) exercise with interval training for the treatment of health related physical fitness components such as cardiovascular endurance, body fat composition, flexibility, muscle strength, muscle endurance and total fitness score among overweight adults with non-communicable disease. The intervention exercise program is a combination of long slow distance activity with interval training alternating of low and medium intensity involving a physical conditioning training with medium volume and low resistant. The study population consisted of twenty-three overweight subjects with 25 to 55 years of age and have a non-communicable disease. Subjects were assigned to a 12-weeks of intervention treatment for 60 min per once, three times in a week with total of 36 sessions. A pretest-posttest research design was used. Statistical analyses include descriptive analysis and paired t-test to determine within group mean differences. Results of the study subject of treatment group experienced a positive improvement in all the six variables after treatment. Results indicate a significant improvement in body composition between pre-test $(\mathrm{M}=33.8)$ and post-test $(\mathrm{M}$ $=30.30$ ). There was a statistical significant increases in cardiovascular endurance, muscle endurance, muscle strength and flexibility. Further to that, fitness score received a significant improvement during pretest $(M=61.13)$ to post-test $(M=65.96)$. Therefore, it is concluded that the intervention of long slow distance exercise with interval training program; had improved all the five health-related physical fitness components and overall fitness score. It was also contributed to a positive finding among overweight adults with the non-communicable disease in Malaysia.
\end{abstract}

Keywords: Health related physical fitness, interval training, long slow distance exercise, non-communicable disease.

*Corresponding author. E-mail: mastura@uniten.edu.my.

Abbreviation: NCD, Non-communicable disease; LCD, long slow distance; ACSM, American College of Sports Medicine.

\section{INTRODUCTION}

In many developing countries, rapid social and economic growth has spurred the emergence ofnon-communicable diseases (NCDs) as a significant public health challenge. This extends to rapidly growing economies like Malaysia, which has witnessed a sharp increase in the prevalence of overweight, obesity, type 2 diabetes mellitus and blood pressure in the past decade (TheThird National Health and Morbidity Survey, 2006). This has contributed significantly to the parallel increase in the prevalence of NCDs (Centers for Disease Control and Prevention, 2016). 
NCD is a medical condition or disease that is not caused by infectious agents (non-infectious or nontransmissible). Non-communicable disease (NCD) can be referred as chronic diseases, which progress slowly and last for long periods. Sometimes, NCD result in premature deaths such as autoimmune diseases, heart diseases, stroke, cancers, diabetes, chronic kidney disease, osteoporosis, Alzheimer's disease, cataracts, and others. NCDs are distinguished only by their noninfectious cause, not necessarily by their duration, though some chronic diseases of long duration may be caused by infections. Chronic diseases require a longer duration of chronic care.

In Malaysia, the annual mortality rate per 100,000 people from non-communicable diseases has increased by $16.7 \%$ since 1990 , an average of $0.7 \%$ a year. A population shift in diet and exercise have led to a dramatic rise in the prevalence of obesity and type 2 diabetes mellitus (Lutsey et al., 2008). The need to promote healthy a lifestyle and work-life balance among adult for the Malaysian population is imperative because the emergence of NCDs transition is now rapidly spreading in Malaysia (Ismail, 2002). Although the trends in overweight and obesity in Malaysia have been summarized in the previous reports (Ismail et al., 2002) an update of obesity prevalence in 2011 and critical appraisal of data on health-related fitness as well as related risk factors are lacking. Patients with NCD like diabetes and high blood pressure have 3 to 4 times higher risk of decreasingphysical fitness and developing depression. At the same time, patients with NCDhave a common physical co-morbid illnesses like cardiovascular diseases, stroke and diabetes. (Ahmad Faudzi et al., 2004).

Recent developments in Malaysia have highlighted the fact that the lack of physical activity or sedentary lifestyle and behavior will not only contribute to the rising obesity rates, but it also directly contributes to the risk of other non-communicable diseases, including high blood pressure, type 2 diabetes, and heart disease (Malaysia Ministry of Health, 2005; USDHHS, 1996, 2000).

The importance of physical activity also contribute to the health of bone, joints, and improved psychological well-being (Ledwidge, 1980; Kahn et al., 2003, Sallis and Owen, 1999). The evidence listed above suggests that the lack of physical activity due to sedentary lifestyle factorswill lead to NCDs. In 2012, it has caused $68 \%$ of all deaths (38 million) which rises from $60 \%$ in 2000 (WHO, 1995). It is to highlight that about half of affected subjects were under the age of 70 and the other half were female. Risk factors such as a person's background, lifestyle and environment increase the likelihood of certain NCDs. Every year, at least 5 million people died because of tobacco use and about 2.8 million died from being overweight. High cholesterol accounts for roughly 2.6 million deaths and 7.5 million deaths because of high blood pressure. This indicates the urgency of comprehensive and collaborative treatment of both physical and mental illnesses. Findings from this study will provide important insights on the design of appropriate intervention programs for the prevention, management, and treatment among overweight subjects with either type 2 diabetes or high blood pressure.

This study investigates and evaluates the effects of the 12 weeks of intervention with the combination of long slow distance (LSD) and interval training exercise regime in the treatment of health-related physical fitness components. Thevariables measured in health-related physical fitness components were cardiovascular endurance, body fat composition, flexibility, muscle strength, muscle endurance and total fitness score among overweight adults suffering non-communicable diseases (type 2 diabetes or high blood pressure). Long slow distance exercise (LSD) is a type of training associated with cardio that involves covering relatively long distance, low intensity and long in duration. While interval training consist of alternating periods of high and low-intensity activity. The intervention of this study combines physical training that includes alternating low and medium intensity of LSD exercise workout and a medium volume and low resistant physical conditioning training with a short rest time that is suitable for sedentary and overweight individuals. Overweight individuals with type 2 diabetes or high blood pressure are at high risk of poor health-related physical fitness and low quality of life, particularly concerning perceived general health and limitations in physical functioning.

Therefore, in this study, the researcher will measure the level of health-related physical fitness components using a comprehensive measurement to assess the positive effect of the intervention. There are two objectives identified in this study as stated below:

i. To investigate the difference between pre-test and posttest scores on health-related physical fitness components namely: Cardiovascular Endurance, Body Fat Composition, Muscle Strength, Muscle Endurance and Flexibility Strength and Total Fitness Score among overweight adults with non-communicable diseases (NCDs).

ii. To identify the difference between pre-test and posttest scores on general health variables namely: body mass index, random blood sugar and blood pressure among overweight adults with non-communicable diseases (NCDs).

\section{MATERIALS AND METHODS}

The study is to observe any beneficial effect of the intervention that combines low slow distance exercise and interval training on twenty-threeoverweight subjects with non-communicable disease (either type 2 diabetes or high blood pressure) on health-related fitness components. The research design used in this study is a quasi-experimental design, pre-test and post-test group designs. Quasi-experiment design provides as much control as possible (Creswell, 2002; Shadish et al., 2002). Comparing pre-test results will indicate degree of equivalency within treatment group 
(Bahaman, 2008). This experimental research attempts to manipulate the independent variable and study the effect of this manipulation or treatment on the dependent variable (Fraenkel and Wallen, 1993).

Physical fitness testing was designed to evaluate and educate participants about the status of their physical fitness. The American Alliance for Health, Physical Education, Recreation and Dance (AAHPERD), and the Cooper Institute for Aerobics Research (CIAR) merged their fitness programs and began to use the same fitness test items and educational materials. American College of Sports Medicine, (ACSM) fitness test consists of five components (ACSM, 2005). In this study, researcher measures the five components of physical fitness, which have been identified as being important because of their relationship to overall health and optimal function. These are body fat composition, flexibility, muscular strength, muscular endurance, and cardiovascular or heart endurance. Health-related physical fitness components were diagnose measuring 5 parameters namely: Cardiovascular Endurance (utilize six minute walk test), Muscle Strength (Five stages sit-up test), Muscle Endurance (utilize the one minute push up test) and Flexibility measurement (using sit and reach test).

Further to that, INBODY machine measure body mass index $\left(\mathrm{kg} / \mathrm{m}^{2}\right)$, percentage of body fat (\%) and fitness score. The accuracy of INBODY 720 was approved by the department of functional anthropology and physiology, faculty of physical culture, University of Palacky' in Olomouc, Czech Republic and Dual Energy X-ray Absorptiometry (DEXA). Random blood sugar test measures the amount of a sugar glucose (mmol/L) and OMRON HEM 7120 Automatic Blood Pressure monitor were utilized to measure blood pressure both systolic and diastolic reading. (Figure 1)

A pre-test was administered to all subjects of this study to measure the health-related physical fitness components parameters before treatment condition was introduced (Figure 2).

However a takeoff pretest was administered to gauge overweight subjects with non-communicable disease either type 2 diabetes or high blood pressure. Subjects were randomly assigned to a 12week of intervention through the combination of long slow distance exercise regime with interval training. The intervention of this study combined physical training that includes alternating of low and medium intensity of long slow distance (LSD) exercise workout and involve a physical conditioning training with medium volume and low resistant of training with a short rest of time.

A pretest-posttest research design were utilized for this study. The twenty-three subjects were measured again at week 12 after the treatment is given (Bordens and Abbot, 1996). Subjects were required to participate in 36 session that comprise 60 minutes for each sessions, 3 times per week for 12 weeks period working at 60 to $70 \%$ of maximum training heart rate. It is gauge bycomparing the differences between pre-test and post-test scores.

Subjects level of health-related physical fitness components measuring 5 parameters were measured at post-test after the 36 session at week 12 to determine the effect of the long slow distance exercise regime program on the dependent variables. Figures 1 and 2 shows treatment assignment and the pre-test-post-test design used in this research study.

For data analysis, mean and standard deviations were used to summarize and describe the mean difference of all the dependent variables of the overweight subjects with non-communicable disease (either type 2 diabetes or high blood pressure) during pretest and post-test (week 12).

The aim at this stage is to describe the general distributional properties of the data, to identify any unusual observations (outliers) or any unusual patterns of observations that may cause problems for later analyses to be carried out on the data and produce plots that visually display distributions of variables (Landau and Everitt, 2004). While statistical analysis, paired sample t-test were used to determine the significant difference between mean scores of two samples within group mean difference (Fraenkel and

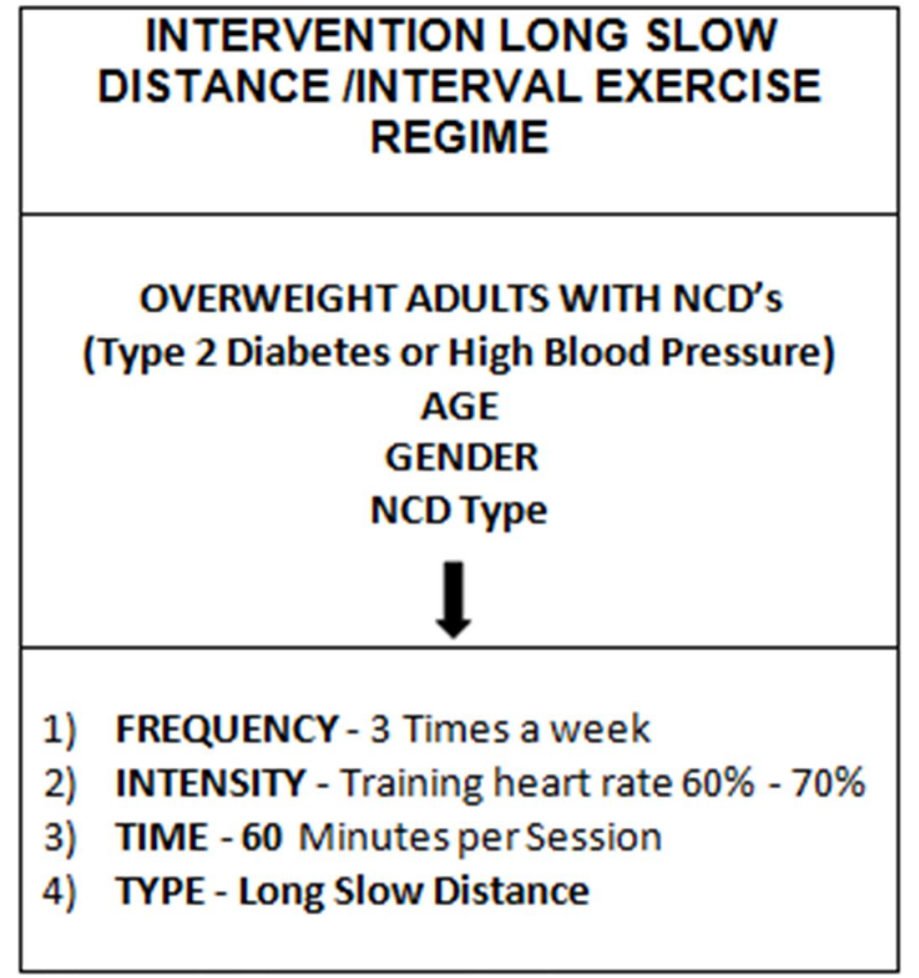

Figure 1. Treatment assignment on independent variable.

Wallen, 1993).

\section{RESULTS}

The results obtained from the preliminary analysis of the frequency distribution are shown in Table 1. Twenty-three subjects were assigned in the treatment of long slow distance exercise intervention. Before the statistical analysis, the respondent profile data was examined. As depicted in Table 1, 17.4\% subjects were male while $82.6 \%$ were female.

Age group was categorized according to three ranges where results indicate that age range 25 to $35,39 \%$, age 36 to 45 were the majority subjects, $52.2 \%$ and age range of 46 to 55 , were $8.8 \%$. From the total number of subjects involved in this research, 39.1\% suffered Type 2 Diabetes, and $60.9 \%$ subjects have high blood pressure.

\section{Statistic data analysis}

\section{Physical fitness for health-components}

In response to the research objective:

To identify the difference between pre-test and post-test scores on health-related physical fitness components namely: Cardiovascular Endurance, Body Fat Composition, Muscle Strength, Muscle Endurance, 


\begin{tabular}{|c|c|c|}
\hline \multicolumn{3}{|c|}{ QUASI-EXPERIMENTAL DESIGN, PRE-TEST AND POST-TEST DESIGNS } \\
\hline $\begin{array}{c}\text { GROUP } \\
\text { TREATMENT } \\
(n=23)\end{array}$ & $\begin{array}{c}\text { PRE-TEST } \\
\text { Week } 1 \\
\text { Session } 1\end{array}$ & $\begin{array}{l}\text { POST-TEST } \\
\text { Week } 12 \\
\text { Sessions } 36\end{array}$ \\
\hline $\begin{array}{l}\text { TREATMENT: } \\
\text { Long Slow Distance/lnterval Training } \\
\text { Exercise Regime } \\
\text { TRAINING HEART RATE: } \\
60-70 \% \text { THR } \\
\text { MUSIC TEMPO } \\
130-145 \text { BPM }\end{array}$ & $\begin{array}{l}\text { Measure } 1 \\
\text { 1. Physical P } \\
\text { 2. INBODY } \\
\text { 3. Blood Pre } \\
\text { 4. Random } \\
\text { 5. Demogra } \\
\text { 6. Attendano } \\
\text { 7. Par-Q Tes } \\
\text { 8. Consent }\end{array}$ & $\begin{array}{l}\text { Measure } 2 \\
\text { nents } \\
\text { is } \\
\text { ge }\end{array}$ \\
\hline
\end{tabular}

Figure 2. The pre-test-post-test design research study.

Table 1. Frequency distribution of subject's age category, gender and NCD category.

\begin{tabular}{lcc}
\hline Variables & Frequency & $\%$ \\
\hline Age (years) & 9 & 39 \\
$25-35$ & 12 & 52.2 \\
$36-45$ & 2 & 8.8 \\
$46-55$ & 23 & 100 \\
Total & & \\
& & \\
Gender & 4 & 17.4 \\
Male & 19 & 82.6 \\
Female & 23 & 100 \\
Total & & \\
& & \\
NCD category & 9 & 39.1 \\
Type 2 diabetes & 14 & 60.9 \\
High blood pressure & 23 & 100 \\
Total & & \\
\hline
\end{tabular}

Flexibility Strength and Fitness score among adults suffering from NCDs.

A paired t-test was conducted to evaluate the impact of the intervention on participant's physical fitness for health components variables. There was a statistical significant improvement in body composition during pre-test $(\mathrm{M}=$ 33.8, $S D=1.609)$ to post-test $(M=30.30, S D=1.031) t$ $(22)=3.168, p<0.05$. There was a statistical significant increase in cardiovascular endurance during pre-test (M $=15.75, \mathrm{SD}=1.25)$ to post-test $(\mathrm{M}=24.25, \mathrm{SD}=1.996) \mathrm{t}$ $(22)=1.352, p<0.05$. Moreover, there was a statistical significant improvement in muscle endurance during pretest $(M=.12 .35, S D=1.460)$ to post-test $(M=33.75 S D$ $=1.712) \mathrm{t}(22)=.658, \mathrm{p}<0.05$ and muscle strength $(\mathrm{M}=$ .050 . $\mathrm{SD}=.2236)$ to post-test $(\mathrm{M}=.350 \mathrm{SD}=.4890) \mathrm{t}$ $(22)=3.19, \mathrm{p}<0.05$. There was also an improvement in flexibility during pre-test $(\mathrm{M}=42.80, \mathrm{SD}=2.015)$ to posttest $(\mathrm{M}=49.85 \mathrm{SD}=1.631) \mathrm{t}(22)=3.704, \mathrm{p}<0.05$. Additionally, fitness score received a significant improvement during pre-test $(\mathrm{M}=.12 .35, \mathrm{SD}=1.460)$ to post-test $(\mathrm{M}=33.75 \mathrm{SD}=1.712) \mathrm{t}(22)=.658, \mathrm{p}<0.05$. All the five components of physical fitness variables received a significant improvement after 12 weeks of treatment through the intervention of the combination of long slow distance with interval training and received high correlation $>.95$. Table 2 described the results clearly.

\section{Non-communicable disease}

In response to the research objective:

To identify the difference between pre-test and post-test scores on general health variables namely: body mass index, random blood sugar and blood pressure among overweight adults with NCDs.

A paired t-test was conducted to evaluate the impact of the intervention on subject's random blood sugar and blood pressure measurement variables. There was a statistical significant improvement in both measurementof the random blood sugar and blood pressure.

There was a significant improvement in body mass index during pre and posttest $(M=30.65, S D=5.68)$ and $(\mathrm{M}=28.86, \mathrm{SD}=5.25) \mathrm{t}(22)=4.84, \mathrm{p}<0.05$. There was 
Table 2. Results of paired t-test during pre-test and post-test on physical fitness for health components scores.

\begin{tabular}{|c|c|c|c|c|c|c|c|}
\hline Physical fitness for health & $\mathbf{N}$ & Mean & & Std. deviation & $t$ & df & Sig \\
\hline \multirow{2}{*}{ Body composition } & 23 & Pre & 33.8 & 1.609 & \multirow{2}{*}{3.168} & \multirow{2}{*}{22} & \multirow{2}{*}{$.003^{*}$} \\
\hline & 23 & Post & 30.30 & 1.031 & & & \\
\hline \multirow{2}{*}{ Cardiovascular endurance } & 23 & Pre & 15.75 & 1.251 & \multirow{2}{*}{1.353} & \multirow{2}{*}{22} & \multirow{2}{*}{$.000^{*}$} \\
\hline & 23 & Post & 24.25 & 1.996 & & & \\
\hline \multirow{2}{*}{ Muscle endurance } & 23 & Pre & 12.35 & 1.460 & \multirow{2}{*}{.658} & \multirow{2}{*}{22} & \multirow{2}{*}{$.000^{*}$} \\
\hline & 23 & Post & 33.75 & 1.712 & & & \\
\hline \multirow{2}{*}{ Muscle strength } & 23 & Pre & .050 & 2236 & \multirow{2}{*}{3.19} & \multirow{2}{*}{22} & \multirow{2}{*}{$.005^{\star}$} \\
\hline & 23 & Post & .350 & .4890 & & & \\
\hline \multirow{2}{*}{ Flexibility } & 23 & Pre & 42.80 & 2.015 & \multirow{2}{*}{3.704} & \multirow{2}{*}{22} & \multirow{2}{*}{$.001^{*}$} \\
\hline & 23 & Post & 49.85 & 1.631 & & & \\
\hline \multirow{2}{*}{ Total fitness score } & 23 & Pre & 61.13 & 7.337 & \multirow{2}{*}{13.23} & \multirow{2}{*}{22} & \multirow{2}{*}{$.000^{*}$} \\
\hline & 23 & Post & 65.956 & 7.182 & & & \\
\hline
\end{tabular}

Note: * The mean difference is significant at the .05 level.

Table 3. Results of paired t-test during pretest and posttest on random blood sugar and blood pressure scores.

\begin{tabular}{|c|c|c|c|c|c|c|c|}
\hline Variables & Group & $\mathbf{N}$ & $\mathbf{M}$ & SD & $t$ & df & Sig (2 tail) \\
\hline \multirow{2}{*}{ Body Mass Index } & Pre & 23 & 30.65 & 5.68 & \multirow{2}{*}{4.84} & \multirow{2}{*}{22} & \multirow{2}{*}{$.000^{*}$} \\
\hline & Post & 23 & 28.86 & 5.25 & & & \\
\hline \multirow{2}{*}{ Random blood sugar (mmol/L) } & Pre & 9 & 7.67 & .590 & \multirow{2}{*}{3.930} & \multirow{2}{*}{8} & \multirow{2}{*}{$.029^{\star}$} \\
\hline & Post & 9 & 6.58 & .492 & & & \\
\hline \multicolumn{8}{|l|}{ Blood pressure } \\
\hline \multirow{2}{*}{ Systolic } & Pre & 14 & 141.6 & 9.781 & \multirow{2}{*}{231} & \multirow{2}{*}{13} & \multirow{2}{*}{$.030^{*}$} \\
\hline & Post & 14 & 133.7 & .9 .067 & & & \\
\hline \multirow{2}{*}{ Diastolic } & Pre & 14 & 88.70 & 6.061 & \multirow{2}{*}{.201} & \multirow{2}{*}{13} & \multirow{2}{*}{$.007^{\star}$} \\
\hline & Post & 14 & 84.60 & 4.935 & & & \\
\hline
\end{tabular}

Note:* The mean difference is significant at the .05 level.

also an improvement in random blood sugar during pretest $(\mathrm{M}=7.67, \mathrm{SD}=.590)$ to post-test $(\mathrm{M}=6.58, \mathrm{SD}=$ $.492) \mathrm{t}(8)=3.90, \mathrm{p}<0.05$. Interestingly, there was a statistical significant improvement in blood pressure measurement systolic pre-test $(\mathrm{M}=.141 .6, \mathrm{SD}=.9 .781)$ to post-test $(\mathrm{M}=.133 .7 \mathrm{SD}=9.067) \mathrm{t}(13)=.231, \mathrm{p}<$ 0.05 and diastolic $(\mathrm{M}=88.70 \mathrm{SD}=6.061)$ to post-test $(\mathrm{M}$ $=84.60 \mathrm{SD}=4.935) \mathrm{t}(13)=.201, \mathrm{p}<0.05$. Table 3 described the results clearly.

\section{DISCUSSION}

The findings of the study revealed that overweight adult subjects with type 2 diabetes or high blood pressure in the higher learning organization with NCDs manifest a statistically significant major effect on the dependent variables of physical fitness components and general health. The result significantly showed $p<0.05$ after undergoing treatment of the intervention through combination of long slow distance with interval training exercise regime for 12 weeks.

Long slow distance and interval training have a similar effect upon aerobic endurance. The changes in maximum oxygen uptake are the same with both types of training when the total work contributed by the subjects are equal. Long slow distance and interval training have also shown to produce similar increases in leg speed (Watson et al., 
1988).

In interval training, a large number of variables can be manipulated in order to produce different types of program. The subjects' heart rate at the end of the work interval is often used as a guide. The next variable that needs to be considered is the duration relief interval. It has to be long enough to allow regeneration of the ATP/CP stores - 20 to 30 seconds is approximately the minimum time required. Useful gains in aerobic endurance can be acquired in 6-8 weeks (Watson et al., 1988). In long slow distance training, the effects on the improvement of aerobic capacity are mainly to increase the general load tolerance and to improve recovery after strenuous exercise (Harre, 1982). Sedentary adult who wishes to improve his general physical conditionmay requiresuch program. Any type of exercise can be used as long as it involves large muscle groups and produces a heart rate in the range of 120 to 150 beats per minute. Gains in aerobic fitness have been reported using programmers of walking, running, skipping, cycling and swimming. Training should occur approximately three times per week because a higher frequency may increase the risk of injury (Watson et al., 1988). The advantages of long slow distance training include that it is only necessary to exercise at least $20 \mathrm{~min}$ at or above threshold heart rate, it can be done with activities such as swimming, cycling and rope skipping and it is sub maximum. It can also be engaged in for a considerable time in relative comfort and is suitable for people just beginning an exercise program. (Sturgeon and Meer, 2006). Long slow distance training is very likely to be the best type of training for very long events. An increasing proportion of fats are oxidized as the duration of activity increases thus sparing 18 the muscle glycogen reserves. The concentration of enzymes involved in fatty acid oxidation is increased by this type of training and the body must also adapt to cope effectively with the dissipation of heat. It is sometimes said that slow, long duration exercise achieves greater capillarisation of muscle. There appears to be no studies which indicate how long duration of training is superior to other types in this respect.

The present study examined the effects of 12 weeks of long slow distance with interval training on health-related physical fitness components among overweight adults with NCD in higher learning organization. The major findings were that the intervention exercise regimes caused large improvements in all the five components of health related fitness. Health-related physical fitness has been defined as specific components of physical fitness related to body composition, cardiorespiratory fitness, muscular fitness, flexibility and body composition. It is well known that aging causes deterioration of all health components if regular physical activity and an active lifestyle are not maintained during the lifespan. In addition, improving or maintaining physical fitness reduces the risk of all-cause and cardiovascular diseases. Despite the fact that fitness is a strong mortality predictor, many studies fail to take into account overall fitness status as an independent risk factor compared to parameters such as hypertension, diabetes, smoking or obesity. Several studies argue that moderate-intensity continuous exercise training (RUN) is an efficient intervention for improving cardiorespiratory fitness regardless of age, training status or gender

The findings of the present study are important and supported by Ross et al. (2000) examined on the short and long-term changes in obesity diagnose which include $\mathrm{BMI}$, and body composition, produced by diet combined with structured aerobics exercise. Sixteen-week randomized controlled trial with a 1-year follow-up, forty obese women (mean body mass index $32.9 \mathrm{~kg} / \mathrm{m}^{2}$; mean weight, $89.2 \mathrm{~kg}$ ) with a mean age of 42.9 years (range, 21-60 years) improved their BMI with a structured aerobics exercise or with the combination of low-fat diet of about $1200 \mathrm{kcal}$. This study produced results which corroborated the findings of a great deal of the previous work in this field (Wen et al., 2009; Weinstein et al., 2004). The study examined the effects of aerobics exercise on obesity diagnose on 15-week aerobics dance courses and various fitness parameters in females. Participants included 112 healthy females who's their mean age was $24.13+5.42$ years, weight $55.25+9.22$ $\mathrm{kg}$, height $1.59+0.06 \mathrm{M}$, and body mass index (BMI) $21.99+3.21$. The subjects were randomly divided into two groups: DS group (double bouts of short duration, $\mathrm{n}=$ 46, twice a week, $1 \mathrm{hr} /$ time) and SL group (single bout of long duration, $\mathrm{n}=66$, once a week, 2 hrs/time). Both groups participated in a designed aerobics dance course for 15 weeks. Prior to the course, and at the completion of 15 weeks, fitness tests were administered for BMI, flexibility; muscular strength, muscular endurance, and 20 seconds shuttle run (agility), single foot standing and cardiorespiratory endurance. Both groups improved significantly $(p<.05)$ in flexibility, muscular strength, muscular endurance, agility, and cardiorespiratory endurance, but the SL group improved more than the DS group. However, statistical analysis revealed that the DS exercise improved more than the SL exercise for those with low BMI $(<15.88)$. For those with high BMI (>28.78), the SL exercise was better than the DS exercise. 15week aerobics dance course given significant improves in fitness for all. However, the DS exercise is superior for those with a low BMI and the SL exercise is superior for those with a high BMI which supported the present study improvised similar to the DS (triple bouts of short duration, $n=20$, three times a week, 50 minutes/time) in the intervention program.

Therefore, from the findings of the study the conclusion that can be drawn is that there were a significant positive effect onphysical fitness components, however there are still no sufficient evidence as to a claim of the superiority of either method in enhancing physical fitness and obesity diagnoseamong overweight adults with NCD's. The researcher therefore, recommends that more researches to be carried out using larger samples and 
suggest pre-test and post-test control group research designs. Despite many benefits observed in this study, there are few limitations. Firstly, the majority of subjects were female $(82 \%)$ while male adults were only $(17 \%)$ aged between 20 and 55 years Secondly, only healthrelated physical fitness components were assessed. Thirdly, we compared long slow distance with interval training only with continuous moderate-intensity training despite that for some fitness components other conventional training programs are much better. Future studies should consider effects of long slow distance compared to other conventional training programs such as high interval training, strength training as well as concurrent training. Also, further research are required to investigate whether long slow distance is suitable for long term maintenance of physical fitness components and whether is it possible to reduce the number of training session without marked decreases of performance elicited by previous aerobics interventions. Finally, effects of long slow distance with interval training on skill related physical fitness components are still unclear.

\section{CONCLUSION}

The intervention of long slow distance exercise with interval training provide an effective and efficient program for overweight and obese individual specifically subject with NCD. Long slow distance running can be a great choice for beginners, injury-prone runners and those just looking to exercise without chasing any time goals. Physical fitness for health components is the best indication of overall fitness, and the combination of long slow distance exercise with interval training dramatically improves recovery and it improved physical fitness on NCD's disease individuals. Moreover, LSD exercise will enhance body's capacity to deliver oxygen to muscles and our body's ability to burn fat as a source of energy as LSD exercise teaches our body to store more energy as glycogen in your muscles. Lastly, LSD exercise is a type of training uses a greater percentage of fat calories than higher intensity activity. LSD exercise helps with weight loss and also acclimates the muscles, bones and joints, preparing the body for higher intensity training. Low intensity, longer duration training is effective in stimulating physiological adaptations and should not be viewed as wasted training time. Over a broad range, increases in total training volume correlate well with improvements in physiological variables and performance.

\section{ACKNOWLEDGEMENT}

This research was supported by UNITEN Internal Grant Scheme (UNIIG 17) Universiti Tenaga Nasional granted to Assoc Prof Dr. Mastura Johar and team.

\section{REFERENCES}

Ahmad Faudzi Y, Amal Nasir M, Gurpeet KM, Azahadi O, Vos T, Rao VPC, Begg S, 2004. Malaysian Burden of Disease and Injury Study. Health Prioritization: Burden of Disease Approach. Institute for Public Health. Ministry of Health, Malaysia.

American College of Sports Medicine (ACSM), 2005. Guidelines for exercise testing and prescription (4th ed.). Philadelphia, PA: Lea \& Febiger.

Bordens KS, Abbott BB, 1996. Research design and methods: A process approach (3rd ed.). MV, California: Mayfield Publishing Company.

Centers for Disease Control and Prevention (CDC), 2016. Physical Activity for a Healthy Weight. https://www.cdc.gov/healthyweight/ physical_activity/

Creswell JW, 2002. Research design: Qualitative, quantitative, and mixed methods approaches. Thousand Oaks, CA: Sage Publications.

Fraenkel JK, Wallen NE, 1993. How to design and evaluate research in education (2nd ed.). New York: McGraw-Hill.

Harre DD, 1982. Principles of sports Institute for Public Health (IPH) The Third National Health and Morbidity Survey (NHMS III) 2006, Nutritional Status. Kuala Lumpur: Ministry of Health Malaysia; 2006.

Ismail MN, 2002. The nutrition and health transition in Malaysia. Public Health Nutr, 5(1):191-195.

Ismail MN, Chee SS, Nawawi H, Yusoff K, Lim TO, James WPT, 2002. Obesity in Malaysia. Obes Rev, 3: 203-208.

Kahn J, Hessling R, Russell D, 2003. Social support, health, and wellbeing among the elderly: What is the role of negative affectivity? Person Individual Diff, 35(1): 5-17.

Landau S, Everitt BS, 2004. A handbook of statistical analyses using SPSS by Chapman \& Hall/CRC Press LLC.

Ledwidge, B., 1980. Run for your mind: Aerobic exercise as a means of alleviating anxiety and depression. Can J Behav Sci, 12(2): 126-140.

Lutsey PL, Steffen LM, Stevens J, 2008. Dietary intake and the development of the metabolic syndrome: the Atherosclerosis Risk in Communities study. Circulation, 117(6): 754-761.

Ministry of Health, 2005. NCD Risk Factors in Malaysia. Disease Control Division, -1 2005/2006.

Ross R, Dagnone D, Jones PJ, Smith H, Paddags A, Hudson R, Janssen I, 2000. Reduction in obesity and related comorbid conditions after diet-induced weight loss or exercise-induced weight loss in men. A randomized, controlled trial. Ann Intern Med, 133: 92103.

Sallis JF, Owen N., 1999. Physical activity and behavioral medicine. Thousand Oaks, California: Sage.

Shadish WR, Cook TD, Campbell DT, 2002. Experimental and quasiexperimental designs for generalized causal inference. Boston: Houghton-Mifflin. An update of a classic by a third author.

Sturgeon J, Meer J, 2006. The first fifty years 1956-2006. President's councilon physical fitness and sport, 40-64. Retrieved from http://fitness.gov/pdfs/50-year-anniversary-booklet.pdf.

Watson D, Clark LA, Tellegen A, 1988. Development and validation of brief measures of positive and negative affect: the PANAS scales. J Pers Soc Psychol, 54(6): 1063-1070.

Weinstein AR, Sesso HD, Lee IM; Cook NR, Manson JE, Buring JE, Gaziano JM, 2004. The relationship of physical activity vs body mass index with type 2 diabetes in women. JAMA; 292(10): 1188-1194.

Wen ZP, Zhou XQ, Feng L, Jiang J, Liu Y, 2009. Effect of dietary pantothenic acid supplement on growth, body composition and intestinal enzyme activities of juvenile Jian carp (Cyprinus carpio var. Jian). Aquaculture Nutr, 15: 470-476.

World Health Organization (WHO), 1995. Committee on physical Activity for health:Exercise for Health, WHO/FIMS Update/Le Point: Bulletin, 73 .

Citation: Johar M, Hung TC, Taha HM, Adnan AR, Ishak MS, Philip A, Azmi MN, 2018. Effect of long slow distance exercise regime on health-related physical fitness components among overweight adults with non-communicable diseases. Int Res J Med Med Sci, 7(1): 9-15. 